\title{
The Interface Region Imaging Spectrograph for the IRIS Small Explorer Mission
}

\author{
Jean-Pierre Wülser*a , Alan M. Title ${ }^{\mathrm{a}}$, James R. Lemen ${ }^{\mathrm{a}}$, Bart De Pontieu ${ }^{\mathrm{a}}$, Charles C. Kankelborg ${ }^{\mathrm{b}}$, \\ Theodore D. Tarbell ${ }^{\mathrm{a}}$, Thomas E. Berger ${ }^{\mathrm{a}}$, Leon Golub ${ }^{\mathrm{c}}$, Gary D. Kushner ${ }^{\mathrm{a}}$, Catherine Y. Chou ${ }^{\mathrm{a}}$, \\ Isaac Weingrod ${ }^{\mathrm{a}}$, Buck Holmes ${ }^{\mathrm{a}}$, Jason Mudge ${ }^{\mathrm{a}}$, William A. Podgorski ${ }^{\mathrm{c}}$ \\ ${ }^{a}$ Lockheed Martin Solar \& Astrophysics Lab, 3251 Hanover St., Palo Alto, CA USA 94304; \\ ${ }^{b}$ Physics Department, Montana State University, Bozeman, MT USA 59717; ${ }^{\mathrm{c} H a r v a r d-S m i t h s o n i a n}$ \\ Center for Astrophysics, 60 Garden Street, MA USA 02138
}

\begin{abstract}
The Interface Region Imaging Spectrograph (IRIS) is a NASA SMall EXplorer mission scheduled for launch in January 2013. The primary goal of IRIS is to understand how the solar atmosphere is energized. The IRIS investigation combines advanced numerical modeling with a high resolution UV imaging spectrograph. IRIS will obtain UV spectra and images with high resolution in space $(0.4 \mathrm{arcsec})$ and time $(1 \mathrm{~s})$ focused on the chromosphere and transition region of the Sun, a complex interface region between the photosphere and corona. The IRIS instrument uses a Cassegrain telescope to feed a dual spectrograph and slit-jaw imager that operate in the $133-141 \mathrm{~nm}$ and $278-283 \mathrm{~nm}$ ranges. This paper describes the instrument with emphasis on the imaging spectrograph, and presents an initial performance assessment from ground test results.
\end{abstract}

Keywords: UV, Sun, corona, chromosphere, spectroscopy

\section{INTRODUCTION}

The Interface Region Imaging Spectrograph (IRIS) is a NASA SMall EXplorer mission currently under development at the Lockheed Martin Solar and Astrophysics Lab. It is scheduled to be launched in January 2013 into a polar sunsynchronous orbit. Its primary objective is to study the interface between photosphere and corona of the sun and address the following scientific questions:

- Which types of non-thermal energy dominate in the chromosphere and beyond?

- How does the chromosphere regulate mass and energy supply to the corona and heliosphere?

- How do magnetic flux and matter rise through the lower atmosphere, and what role does flux emergence play in flares and mass ejections?

To find answers to these questions, IRIS will trace the flow of energy and plasma through the solar atmosphere using UV spectroscopy and imaging with high angular, temporal, and spectral resolution. IRIS observes two spectral bands: 1 . the vicinity of the chromospheric $\mathrm{Mg}$ II h and k lines between 278.5 and $283.5 \mathrm{~nm}$, and 2. the region of solar emission lines between 133.2 and $140.6 \mathrm{~nm}$ covering a broad range of chromospheric, transition region, and coronal temperatures. Table 1 lists some of the key solar lines covered by IRIS.

Table 1. Key solar lines observed with the IRIS spectrograph. The strongest lines suitable for high cadence observations are marked with an asterisk $(*)$. These lines are also observed by the slit-jaw imager.

\begin{tabular}{|l|c|c|c|c|c|c|c|c|}
\hline Line & O I & Mg II wing* & Mg II h \& k & C II * & Si IV * & O IV & Fe XII & Fe XXI \\
\hline$\lambda(\mathrm{nm})$ & 135.6 & 283 & $279.6,280.3$ & $133.5,133.6$ & $139.4,140.3$ & $140.0,140.1$ & 134.9 & 135.4 \\
\hline LogT $(\mathrm{K})$ & 3.8 & $3.7-3.9$ & 4.0 & 4.3 & 4.8 & 5.2 & 6.2 & 7.0 \\
\hline
\end{tabular}

*wuelser@1msal.com phone: 650-424-3289

Space Telescopes and Instrumentation 2012: Ultraviolet to Gamma Ray,

edited by Tadayuki Takahashi, Stephen S. Murray, Jan-Willem A. den Herder,

Proc. of SPIE Vol. 8443, 844308 - @ 2012 SPIE - CCC code: 0277-786/12/\$18 - doi: 10.1117/12.927036

Proc. of SPIE Vol. 8443 844308-1 
Figure 1 shows the conceptual design of the IRIS instrument. It consists of a $19 \mathrm{~cm}$ aperture Cassegrain telescope that feeds a dual range UV spectrograph and slit-jaw imager. In Figure 1, sunlight enters the telescope from the right. Most of the solar energy then passes through the substrate of the primary mirror, gets absorbed on a plate behind the mirror and is then radiated to space. A dielectric coating on the primary and secondary mirrors primarily reflects the UV wavelengths of interest towards the spectrograph slit. The IRIS telescope and its thermal concept are discussed in more detail elsewhere at this conference $e^{1,2}$.

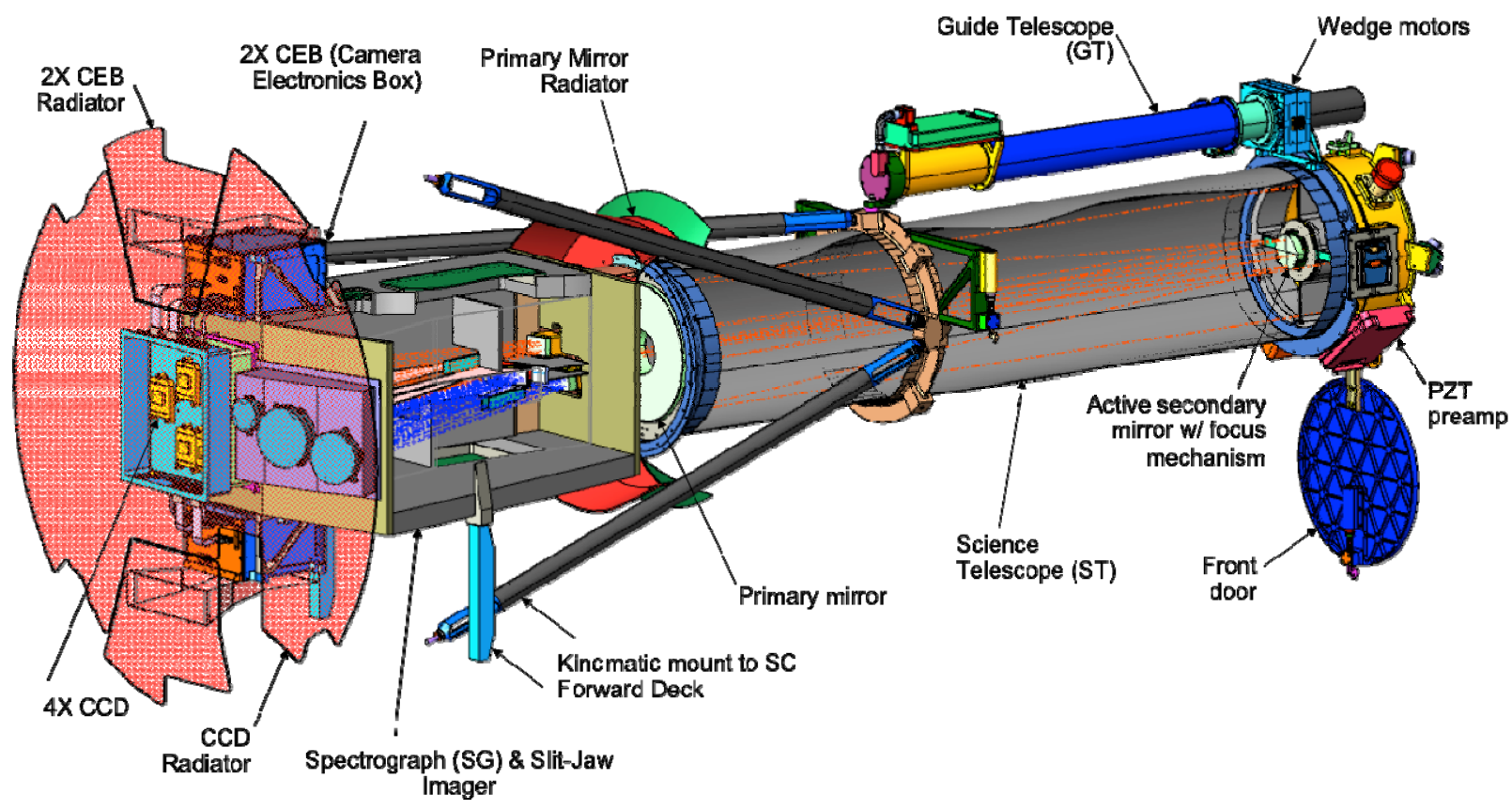

Figure 1. Conceptual design of the IRIS instrument. Sunlight comes in from the right. In the actual instrument, the spectrograph is rotated 180 degrees about the instrument axis relative to the science telescope (see Figure 2).

Figure 2 shows the IRIS instrument after the telescope was integrated with the spectrograph. For accommodation purposes, the spectrograph structure has been rotated 180 degrees about the instrument axis since the conceptual design: the detector housing (silver box on the left) is now facing the viewer. Other components such as the Guide Telescope (GT), the primary mirror radiator, and the Camera Electronics Boxes can be identified by comparing with Figure 1.

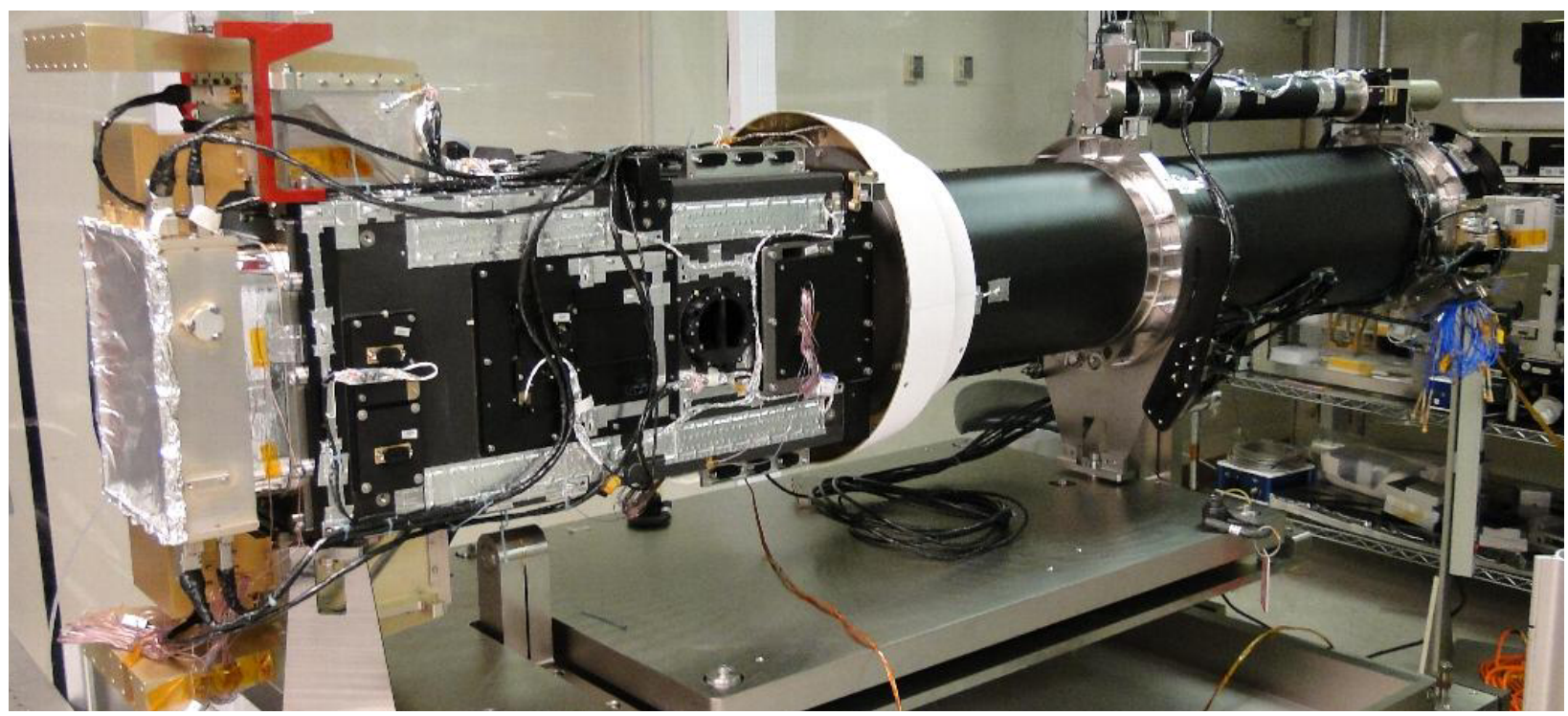

Figure 2. The IRIS instrument after integration of the telescope with the spectrograph. 
IRIS is a single instrument mission on a sun-pointing spacecraft. The instrument GT acts as the spacecraft fine sun sensor. The instrument axis can be pointed to any point on the sun and up to two arcminutes above the solar limb using a pair of motorized glass wedges in front of the GT, just as was done on the TRACE mission. The spacecraft is equipped with two star trackers that control the spacecraft roll and allow for any spectrograph slit orientation during observations. Table 2 summarizes the characteristics of the IRIS instrument and spectrograph / slit-jaw imager.

Table 2. IRIS instrument characteristics

\begin{tabular}{|c|c|c|c|}
\hline & Telescope / instrument & Spectrograph & Slit-jaw imager \\
\hline Optics & Classical Cassegrain & $\begin{array}{l}\text { Dual range Czerny-Turner } \\
\text { w/single slit \& predisperser }\end{array}$ & $\begin{array}{l}\text { Reflective slit-jaws, } \\
\text { re-imaging mirror }\end{array}$ \\
\hline Aperture / effective area & $19 \mathrm{~cm}$ diameter & $\sim 2 \mathrm{~cm}^{2} @ 140 \mathrm{~nm}$, & $\sim 0.7 \mathrm{~cm}^{2} @ 140 \mathrm{~nm}$ \\
\hline Focal length & $6.895 \mathrm{~m}$ & $1.257 \mathrm{~m}$ & $0.933 \mathrm{~m}$ \\
\hline Magnification & 4.9 & 2.33 & 2.33 \\
\hline Spatial resolution / scale & $<0.4 \operatorname{arcsec}$ & $1 / 6 \operatorname{arcsec} /$ pixel & $1 / 6 \operatorname{arcsec} /$ pixel \\
\hline Field of view / slit size & $175 \operatorname{arcsec}$ & $175 \operatorname{arcsec} \times 1 / 3 \operatorname{arcsec}$ & $175 \times 175 \operatorname{arcsec}$ \\
\hline Spatial scan range & & $40 \operatorname{arcsec}$ & \\
\hline Pointing range & \multicolumn{3}{|c|}{$+/-20$ arcmin from Sun center; any slit orientation } \\
\hline Spectral ranges & & $\begin{array}{c}133.2-135.8 \mathrm{~nm}, 139.0-140.6 \mathrm{~nm}, \\
278.5-283.5 \mathrm{~nm}\end{array}$ & $\begin{array}{c}134 \mathrm{~nm}, 140 \mathrm{~nm}, \\
279.6 \mathrm{~nm}, 282.9 \mathrm{~nm}\end{array}$ \\
\hline Spectral scale, bandwidth & & $\begin{array}{l}\text { FUV: } 1.28 \mathrm{pm} / \text { pixel, } \\
\text { NUV: } 2.55 \mathrm{pm} / \text { pixel }\end{array}$ & $\begin{array}{l}\text { FUV: } \sim 5 \mathrm{~nm}, \\
\text { NUV: } 0.38 \mathrm{~nm}\end{array}$ \\
\hline Detector & & $\begin{array}{l}3 \text { CCDs, back illuminated, } \\
1056 \times 2061,13 \mu \mathrm{m} \text { pixels }\end{array}$ & $\begin{array}{l}\text { CCD, back illuminated, } \\
1056 \times 2061,13 \mu \mathrm{m} \text { pixels }\end{array}$ \\
\hline Vis./IR rejection & $\sim 600 \mathrm{x}$ & $\geq 4 \times 10^{8} \times$ & $\geq 4 \times 10^{8} \times$ \\
\hline
\end{tabular}

The remainder of the paper will focus on the spectrograph and slit-jaw imager, and will provide an initial assessment of the overall instrument performance.

\section{SPECTROGRAPH AND SLIT-JAW IMAGER}

\subsection{Overview}

The optical layout of the spectrograph (SG) and the slit-jaw imager (SJI) are shown in Fig. 3. The UV spectrograph is a Czerny-Turner design with a pre-disperser at the slit, and separate optical paths thereafter for the 278.5-283.5 nm nearUV band, and the 133.2-140.6 nm far-UV band, respectively. The slit-jaw imager optics re-image the solar image reflected from the slit jaws onto the SJI CCD. Filters in the filter wheel select one of the two SJI paths for any given exposure: reflection filters feed the FUV path, while transmission filters pass the light to the NUV path. The SG and SJI (as well as the telescope) make extensive use of narrowband dielectric mirror coatings developed by Acton Research Corp. to suppress out-of-band UV, visible, and IR radiation.

All components of the system are located in the spectrograph structure, which is made of aluminum honeycomb panels with graphite epoxy face sheets (Figure 4). 


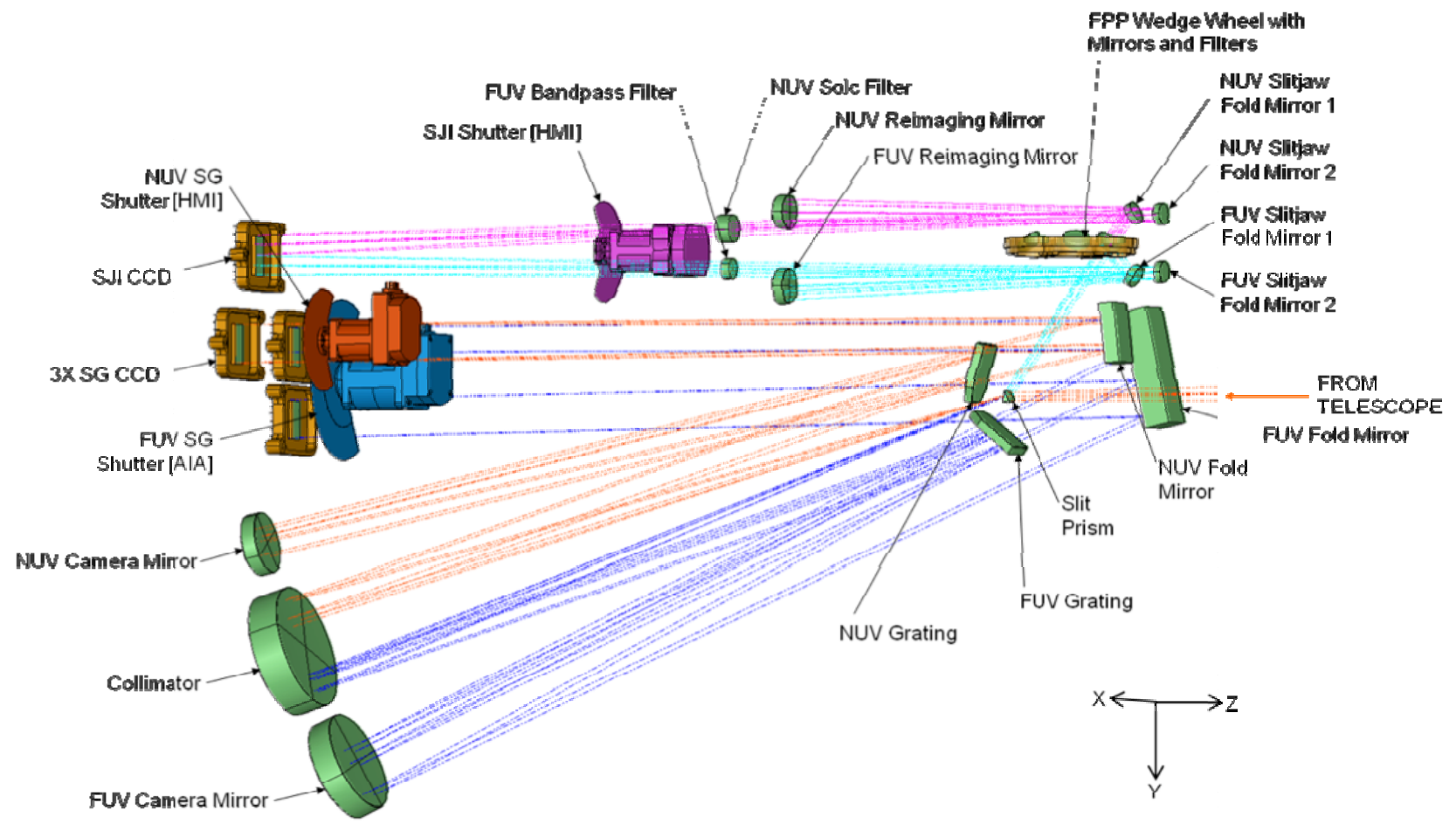

Figure 3. Optical paths in the IRIS spectrograph and slit-jaw imager (SJI). Blue: FUV spectrograph, orange: NUV spectrograph, cyan: FUV SJI, magenta: NUV SJI.

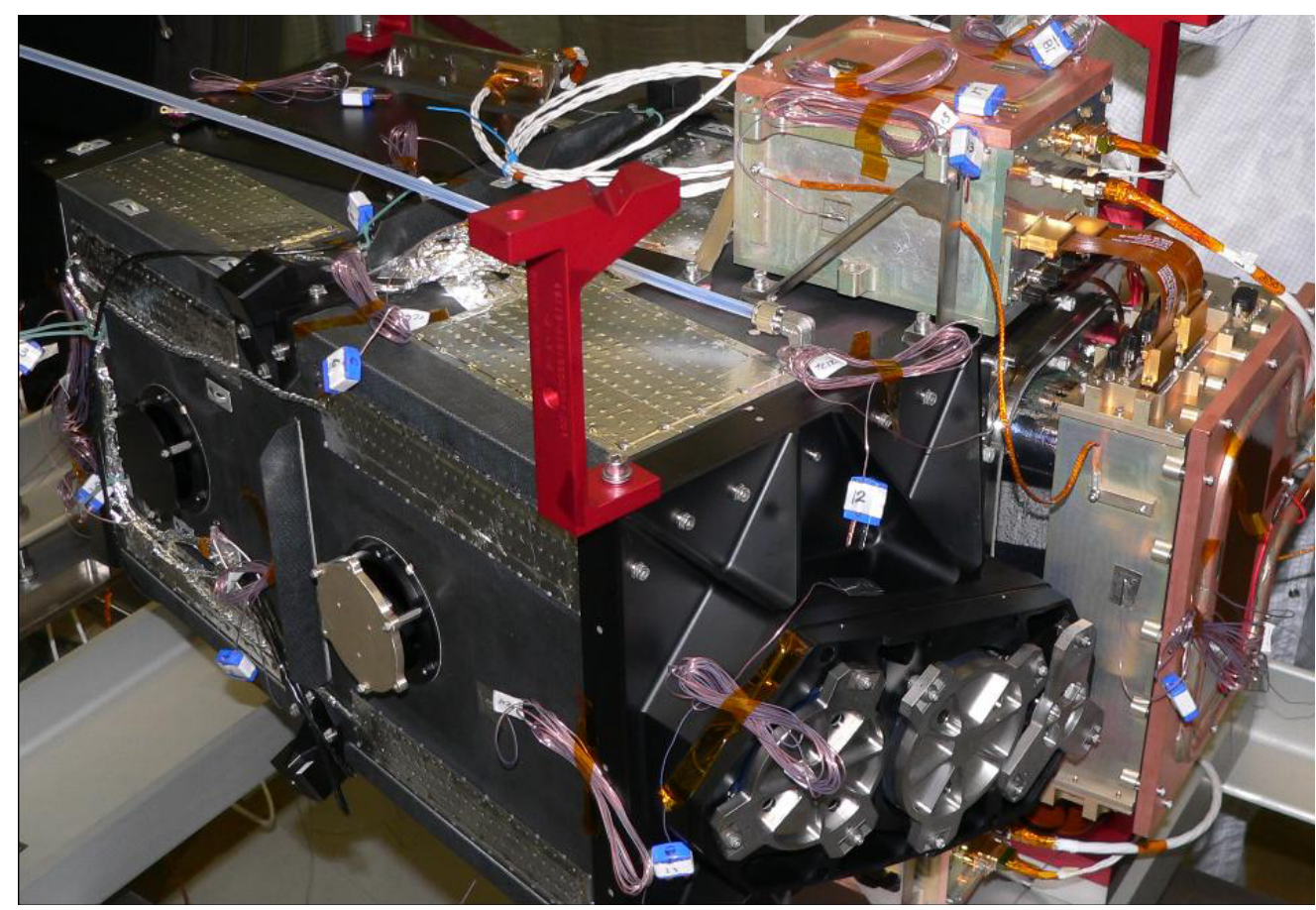

Figure 4. The spectrograph structure in preparation for vacuum testing. The backside of the structure is dominated by the detector assembly on the right that houses all four CCD detectors, and to the left of it, the mounts of the NUV camera mirror, the collimator mirror, and the FUV camera mirror. The rectangular box on the top is one of the two camera electronics boxes. 


\subsection{Spectrograph}

In Figure 3, light from the telescope is focused onto the slit/predisperser, essentially a small $\mathrm{MgF}_{2}$ prism with vapordeposited aluminum slit jaws on the first surface. The latter form the $175 \times 1 / 3$ arcsec spectrograph entrance slit for both wavelength ranges. The $\mathrm{MgF}_{2}$ prism is strongly dispersive between 280 and $140 \mathrm{~nm}$, directing the near-UV light (278.5$283.5 \mathrm{~nm}$, orange rays in Fig. 3) and far-UV light (133.2-140.6 nm, blue rays) onto separate halves of the collimator mirror. Besides physically separating the two UV bands, the predisperser also directs $90-95 \%$ of the residual visible and infrared radiation from the telescope into the near-UV band, thus further reducing the undesired long wavelength contribution in the far-UV band.

The single collimator mirror sends the two spectral bands to separate gratings, camera mirrors, and detectors. The spectrograph gratings, fabricated by Horiba Jobin-Yvon are plane, holographically recorded, and quasi-sinusoidal, with a groove density of 3600 lines $/ \mathrm{mm}$. Figure 5 shows the two gratings in close proximity to the slit prism assembly inside the spectrograph. The two spectral bands each have their own shutter mechanisms (based on SDO AIA ${ }^{3}$ and $\mathrm{HMI}^{4}$ designs). The spectrograph uses three detectors: one CCD covers the NUV band, while two CCDs cover the 133.2135.8 $\mathrm{nm}$ and 139.0-140.6 nm portions of the FUV band, respectively. Up to the gratings, all optical elements and the optical axis are in a single plane. The camera mirrors, fold mirrors, and detectors, however, are out of plane: Figure 3 shows the NUV elements above the main plane and the FUV elements below. This arrangement provides a compact design with all CCD detectors in the same focal plane and near each other (Figure 4).

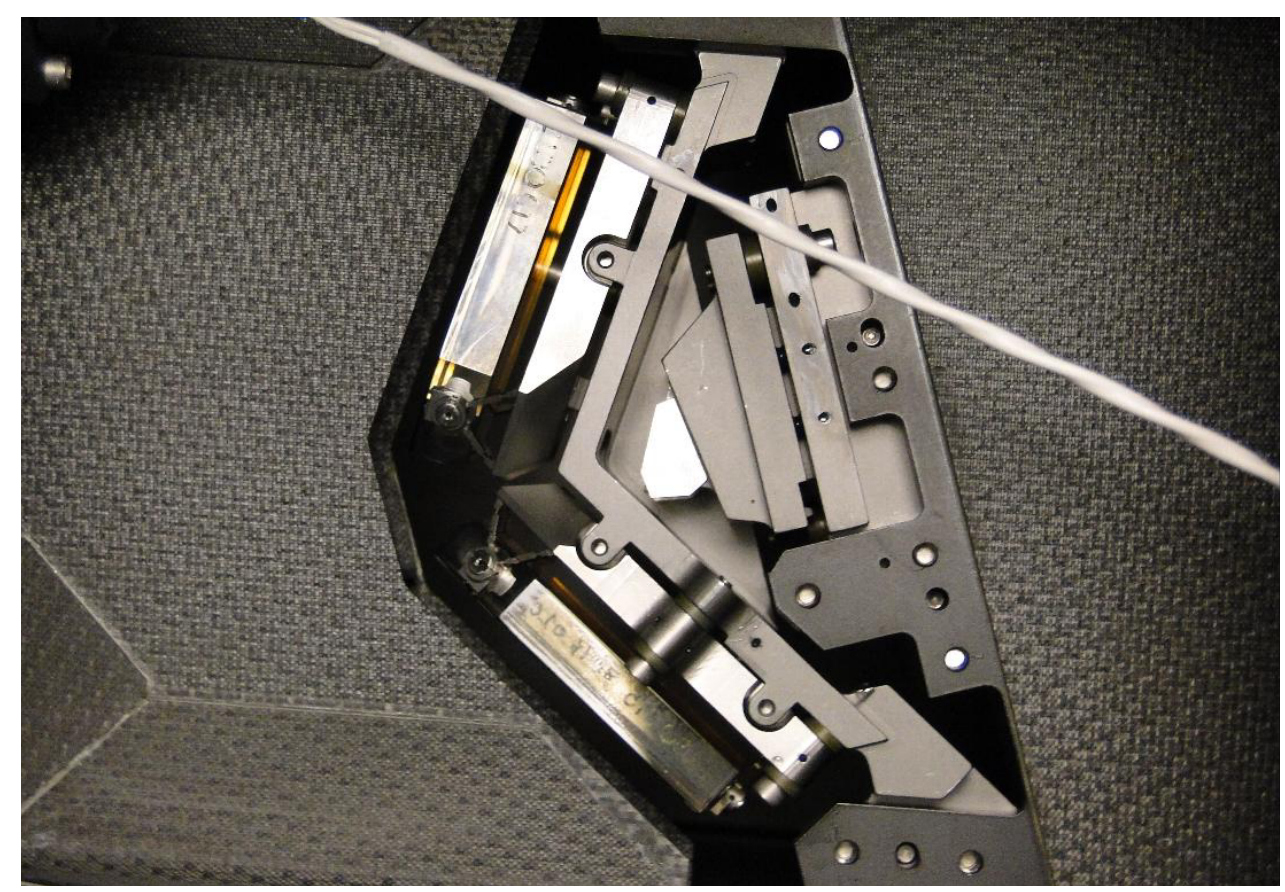

Figure 5. The FUV (top) and NUV gratings in close proximity to the slit prism assembly inside the spectrograph structure. The grating surfaces are perpendicular to the plane of the photo. The slit prism is located behind the tear-drop shaped silver cover at the center of the image.

\subsection{Slit-jaw imager}

The NUV and FUV paths of the slit-jaw imager each have a re-imaging mirror and two fold mirrors, share a common filter wheel, common shutter, and image onto separate halves of the common slit-jaw imager CCD. The FUV path includes a fixed FUV bandpass filter to block longer wavelength light. The NUV path includes a Solc birefringent filter element to reduce the near-UV bandwidth to $0.38 \mathrm{~nm}$ (Figure 6). The filter consists of a stack of 8 Quartz plates of equal thickness, and one wire-grid polarizer at each end of the stack. The filter is encapsulated in a hermetically sealed housing to prevent the index-matching compound from spreading to the contamination sensitive FUV optics. 

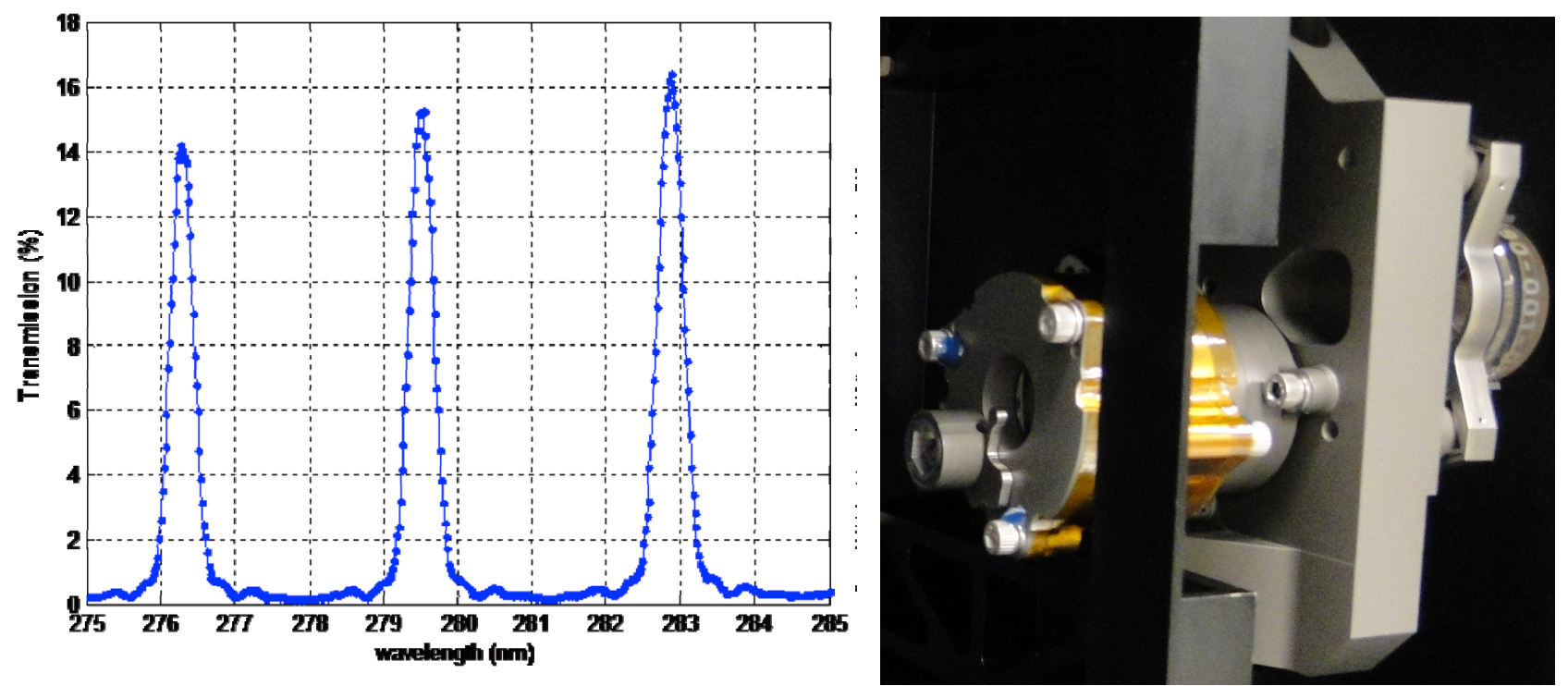

Figure 6. Left: transmission of the Solc birefringent filter. It has a FWHM of $0.38 \mathrm{~nm}$ and a free spectral range of $3.3 \mathrm{~nm}$. Either the peak at $279.6 \mathrm{~nm}$ or the peak at $282.9 \mathrm{~nm}$ is selected via prefilter in the filter wheel. Right: The Solc filter in its sealed housing inside the spectrograph. The edge of the NUV re-imaging mirror is also seen on the far right.

The filter wheel determines the wavelength selection of the slit-jaw imager. It holds two near-UV pre-filters centered on $279.6 \mathrm{~nm}$ and $282.9 \mathrm{~nm}$, respectively that feed the NUV path and the Solc filter. Two mirrors predominantly reflect far-UV radiation around $134 \mathrm{~nm}$ and $140 \mathrm{~nm}$, respectively to the FUV path. Figure 7 shows their reflectivity curves. The remaining two filter wheel positions hold a clear glass element, and an Al-coated mirror for ground testing.

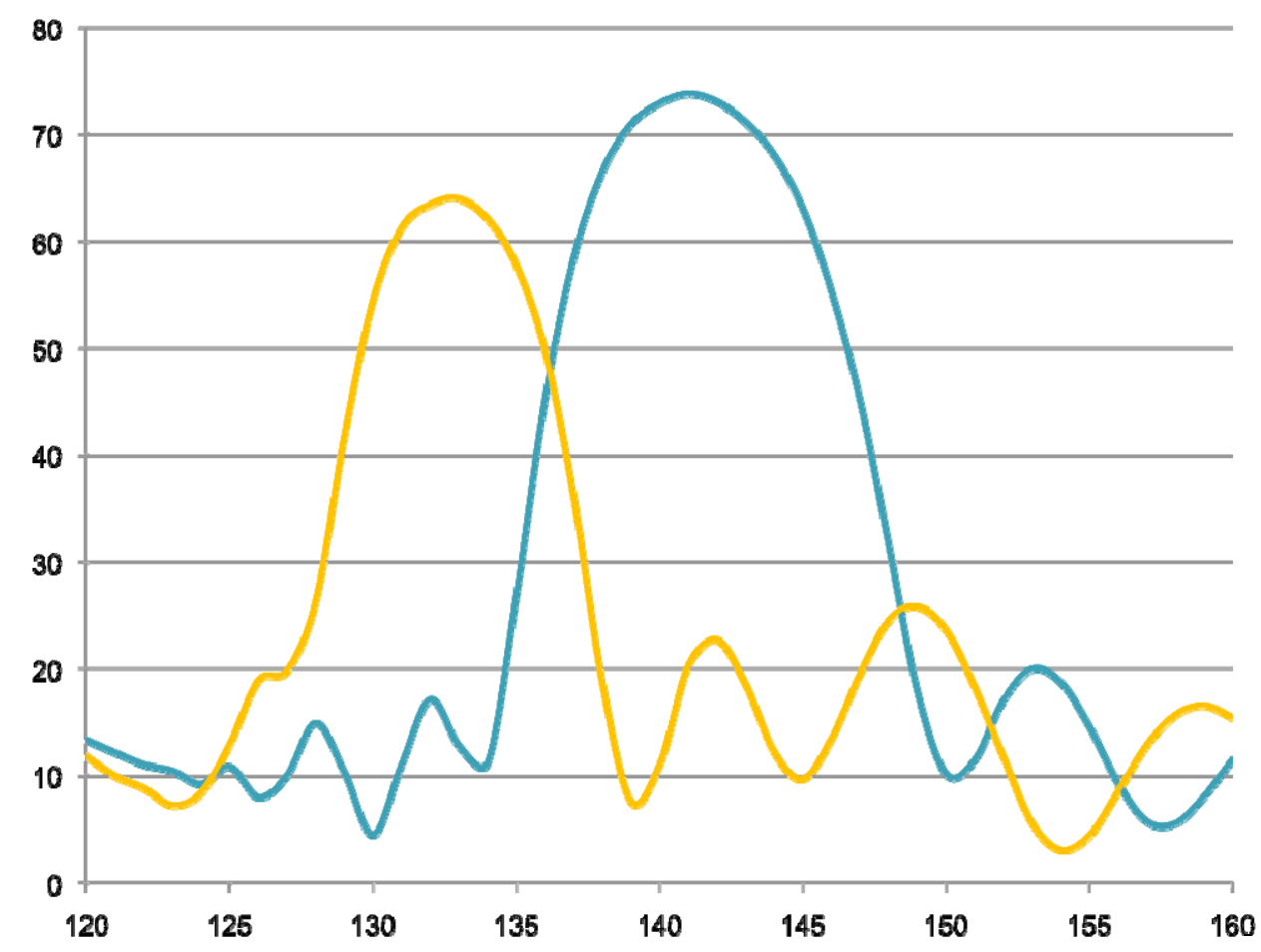

Figure 7. Reflectivity (in \%) as a function of wavelength (in $\mathrm{nm}$ ) for the two FUV filter wheel mirrors. The shorter wavelength mirror predominantly reflects emission from the chromospheric C II lines to the SJI, while the longer wavelength mirror mostly reflects emission from the transition region Si IV lines. The above curves do not include the effect of the coatings of other elements in the system which strongly attenuate radiation below $132 \mathrm{~nm}$, and above $142 \mathrm{~nm}$. 


\subsection{Optics mounts, detectors}

Most of the optics are mounted with flexure mounts. Some of them are shown in Figure 8.

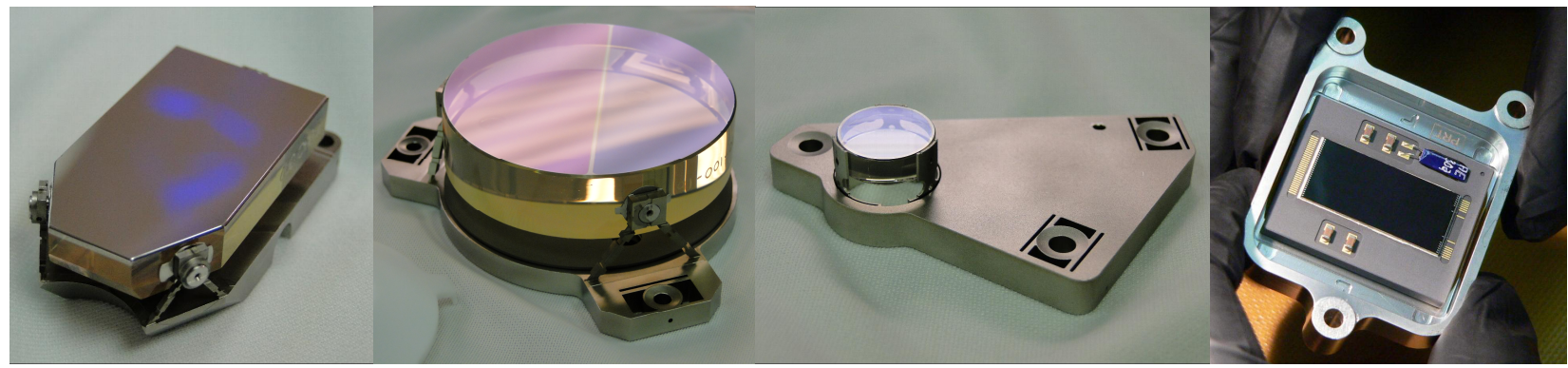

Figure 8. From left to right: mounted grating, FUV collimator, SJI fold mirror, CCD detector.

The four detectors are custom backside illuminated CCD devices manufactured by e2v (Figure 8). All CCDs are identical with $1056 \times 2061$ square 13 micron pixels. The serial register is located on the short side of the CCD, i.e., the parallel transfer occurs in the spectral direction. The CCDs are read out using two flight spare cameras from the SDO AIA and HMI instruments.

\section{INITIAL PERFORMANCE ASSESSMENT}

For much of the integration, alignment, and testing of the spectrograph and SJI, we utilized a custom $279.6 \mathrm{~nm}$ laser provided by Toptica, and UV LEDs. During vacuum testing we illuminated the spectrograph slit with a Deuterium lamp. Here we present some preliminary imaging and spectral measurements from recent spectrograph tests.

\subsection{Spectrograph}

Figure 9 shows the spectrum of a Deuterium lamp observed with the IRIS FUV spectrograph. The top and bottom half of the panel are from the two FUV CCDs. The spectral lines are very sharp, less than 3 pixels or 4 pm wide, illustrating that the spectrograph resolution meets its design goal. The lines are slightly slanted relative to the dispersion direction which will be corrected in post-processing.

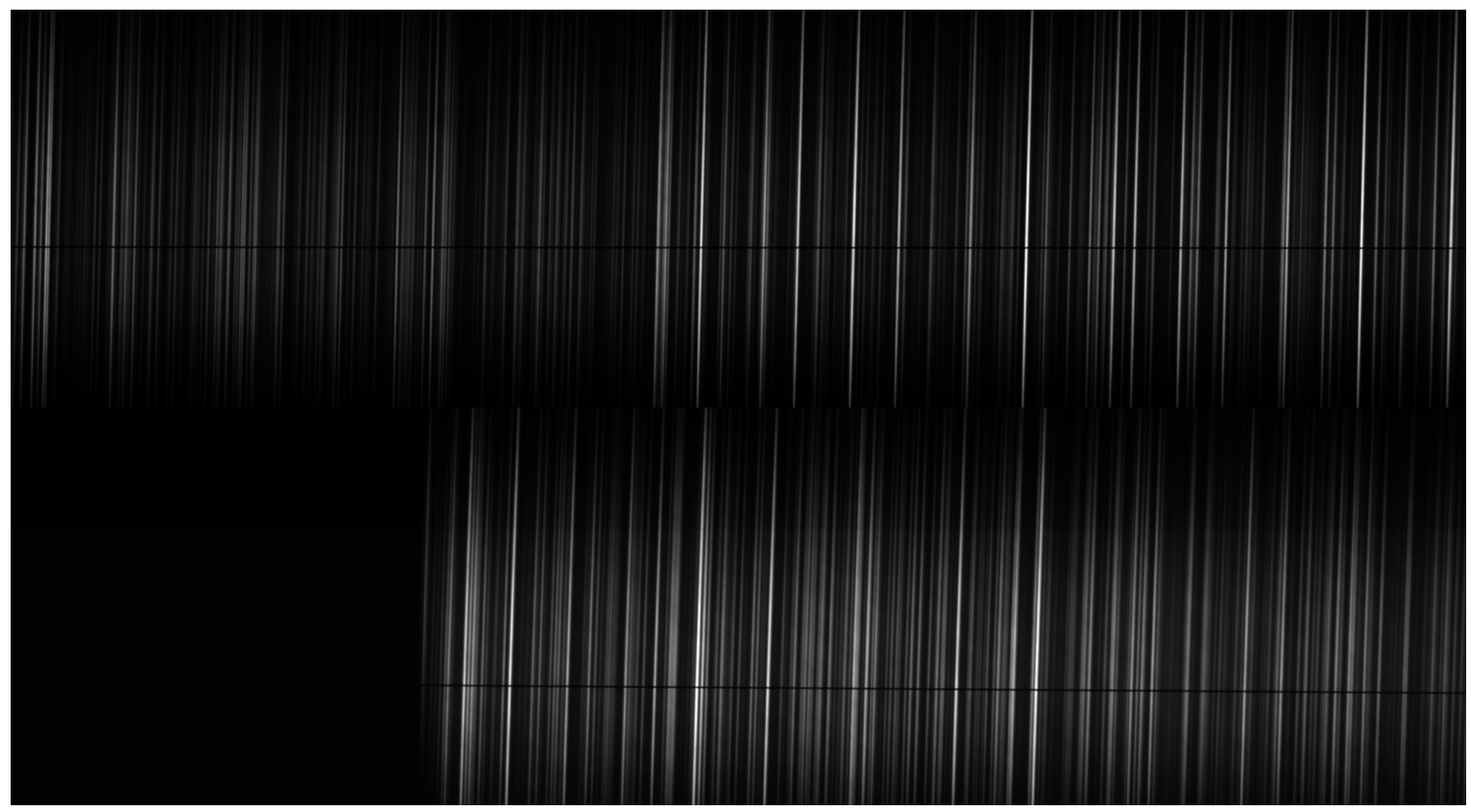

Figure 9. Spectrum of a Deuterium lamp on the two CCDs of the FUV spectrograph (top and bottom half of figure). The spectral ranges are 133.2-135.8 $\mathrm{nm}$ (top) and 138.8-140.7 nm (bottom). The dark horizontal lines are from one of the two fiducial marks on the slit that aid in registering data from the spectrographs with the slit-jaw images. 
Figure 10 shows a $0.8 \mathrm{~nm}$ wide section of the Deuterium lamp spectrum in comparison with a spectrum published by Abgrall et al. (1999) ${ }^{5}$. We use such comparisons to verify our spectral alignment and plate scale.

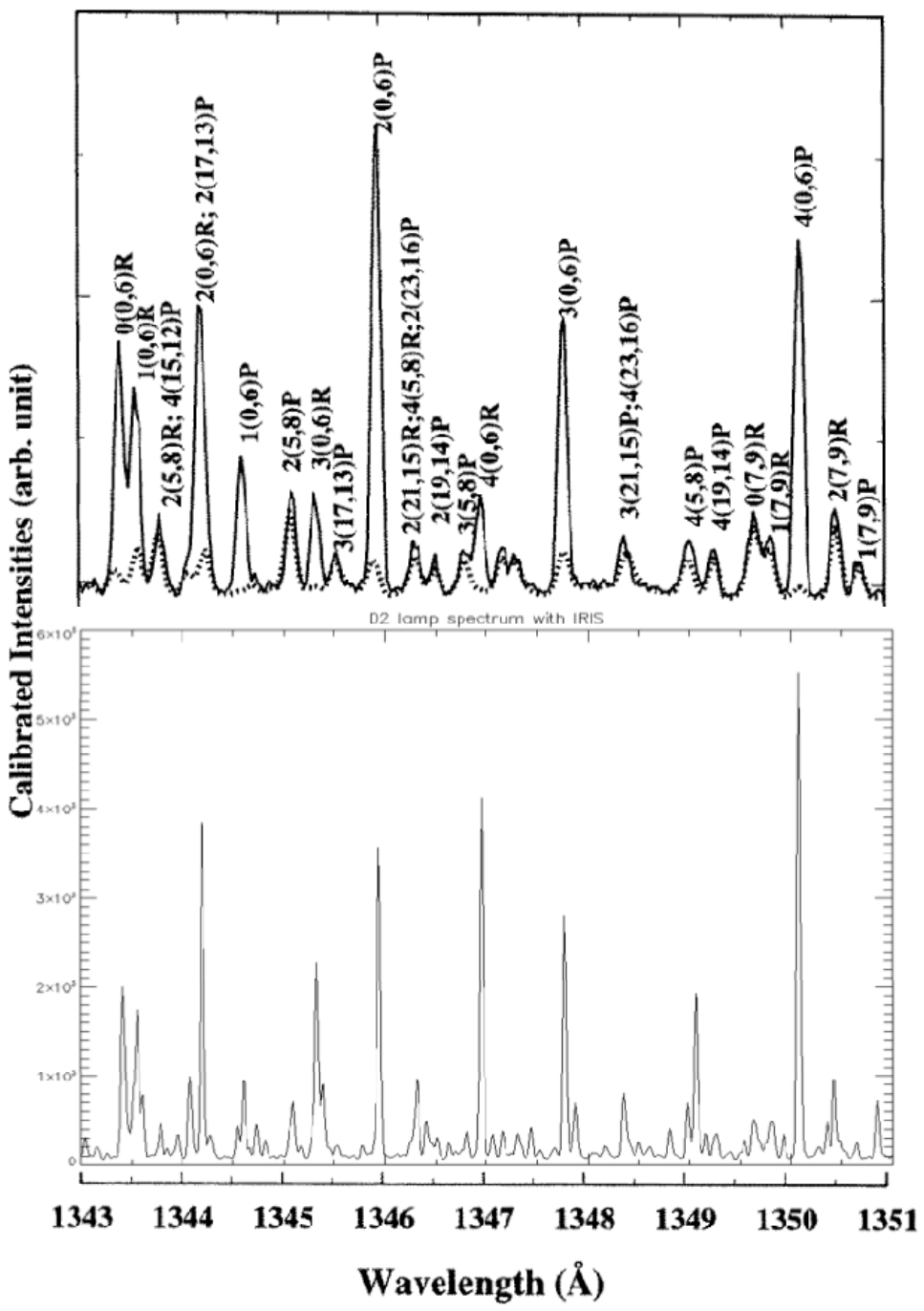

Figure 10. Comparison of a Deuterium spectrum by Abgrall et al. (1999) ${ }^{5}$ (top) and as observed by IRIS (bottom). The relative line intensities are not identical between the two spectra since Abgrall et al. used a different plasma excitation method for their measurements, but the line centroids may be used to calibrate the dispersion of the IRIS FUV spectrograph.

\subsection{Slit-jaw imager}

Figure 11 shows two images of a portion of the slit taken with the FUV and NUV slit-jaw imagers. For the FUV image on the left the slit was illuminated by a Deuterium lamp, for the NUV image on the right, a UV LED was used. The images clearly resolve the $1 / 3$ arcsec wide slit and the fiducial mark.

Both images show a patchwork pattern that results from the process that e2v uses to enhance the UV QE of their backside illuminated CCDs. The pattern is removable via flat-fielding techniques. 

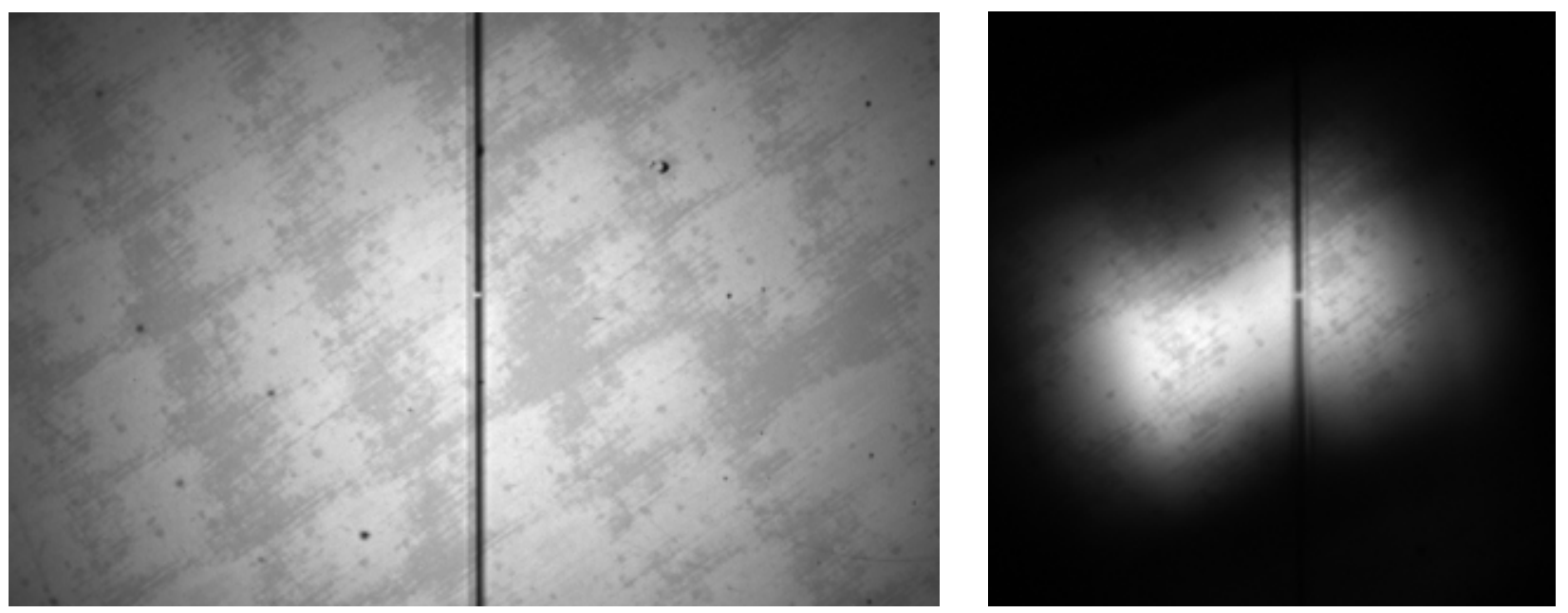

Figure 11. Left: FUV slit-jaw image of the slit with fiducial mark illuminated by a Deuterium lamp. Right: NUV slit-jaw image of the slit illuminated with a $280 \mathrm{~nm}$ UV LED as the source.

\subsection{Instrument spectral response}
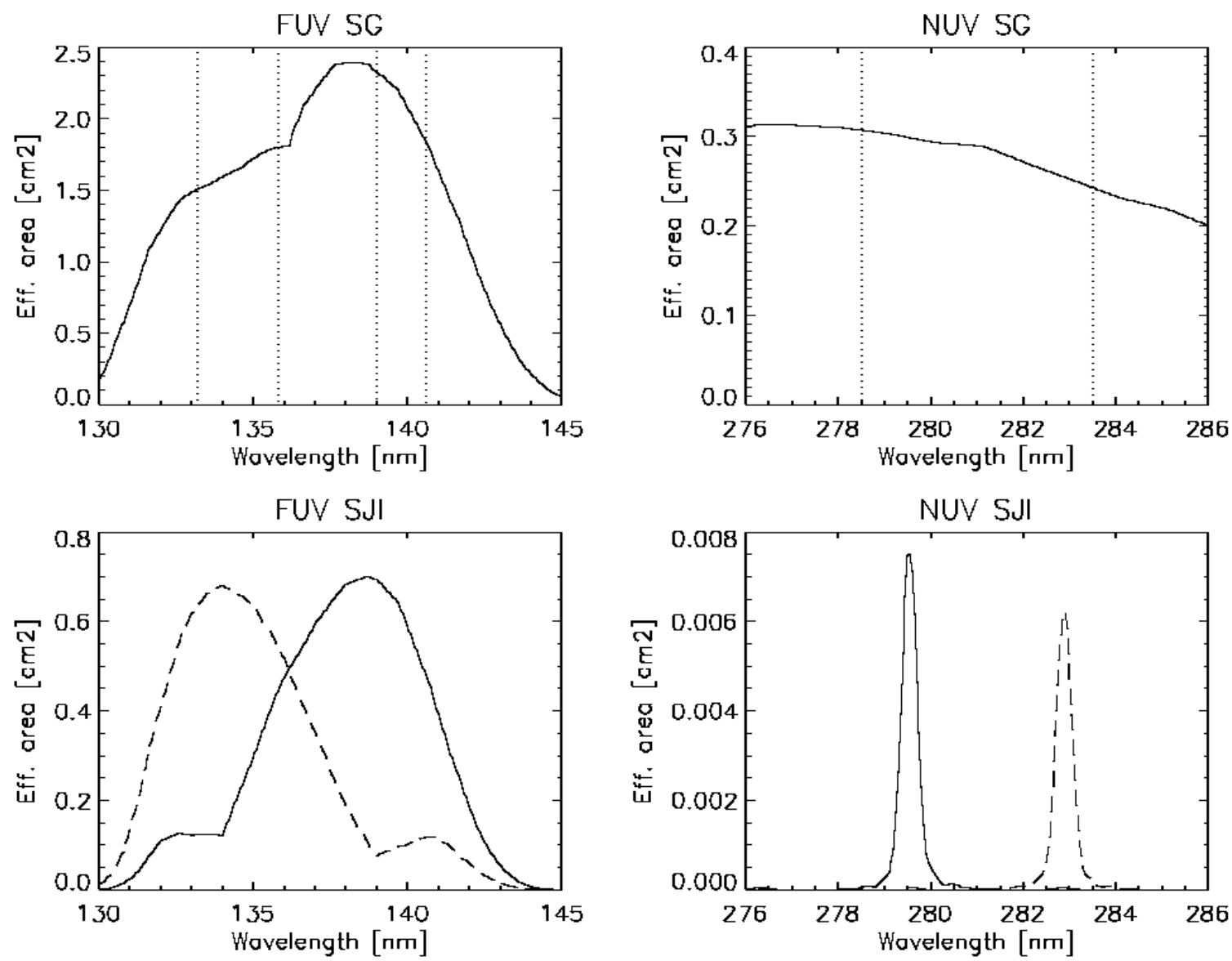

Figure 12. IRIS effective area as a function of wavelength for all spectrograph and slit-jaw imager channels. In the two top panels, the spectrograph spectral ranges are indicated by dotted lines. The spectral response of the slit-jaw imager is shown for each of the two FUV (left) and NUV (right) filter wheel positions using solid and dashed lines. 
Figure 12 shows the overall instrument spectral response (or effective area) for all four spectrograph and slit-jaw imager paths. The curves are based on the product of measurements of the individual optics. The top panels show that the spectrograph responses are sufficiently well-behaved to allow a spectral calibration over their ranges, in spite of the extensive use of narrow-band dielectric mirror coatings. The bottom two panels show the selective response of the filters in the slit-jaw imagers.

\section{SUMMARY AND CONCLUSIONS}

We have presented a summary of the IRIS instrument with emphasis on the spectrograph and slit-jaw imager systems. The IRIS instrument has completed its integration, is currently undergoing comprehensive testing, and is about to be integrated with the spacecraft. Preliminary test results such as those presented in this paper indicate that the instrument is performing very well.

The IRIS mission would not be possible without the efforts of many, including those at our partner institutions at Montana State University, and the Smithsonian Astrophysical Observatory.

\section{REFERENCES}

[1] Park, S. C., Cheimets, P. N., Podgorski, W. A., Bergner, H. W., Yanari, C. and Wuelser, J.-P., "Design of interface region imaging spectrograph (IRIS) primary mirror thermal management system," Proc. SPIE 8443-123 (2012).

[2] Podgorski, W. A., Cheimets, P. N., Golub, L., Lemen, J. R. and Title, A. M., "Design, performance prediction, and measurements of the Interface Region Imaging Spectrograph (IRIS) Telescope," Proc. SPIE 8443-124 (2012).

[3] Lemen, J. R. and 46 co-authors, "The Atmospheric Imaging Assembly (AIA) on the Solar Dynamics Observatory (SDO)," Solar Phys. 275, 17-40 (2012).

[4] Scherrer, P. H. and 12 co-authors, "The Helioseismic and Magnetic Imager (HMI) Investigation for the Solar Dynamics Observatory (SDO)," Solar Phys. 275, 207-227 (2012).

[5] Abgrall, H., Roueff, E., Xianming Liu, Shemasky, D. E. and James, G. K., "High-resolution far ultraviolet emission spectra of electron-excited molecular deuterium," J. Phys. B: At. Mol. Opt. Phys. 32, 3813-3838 (1999). 